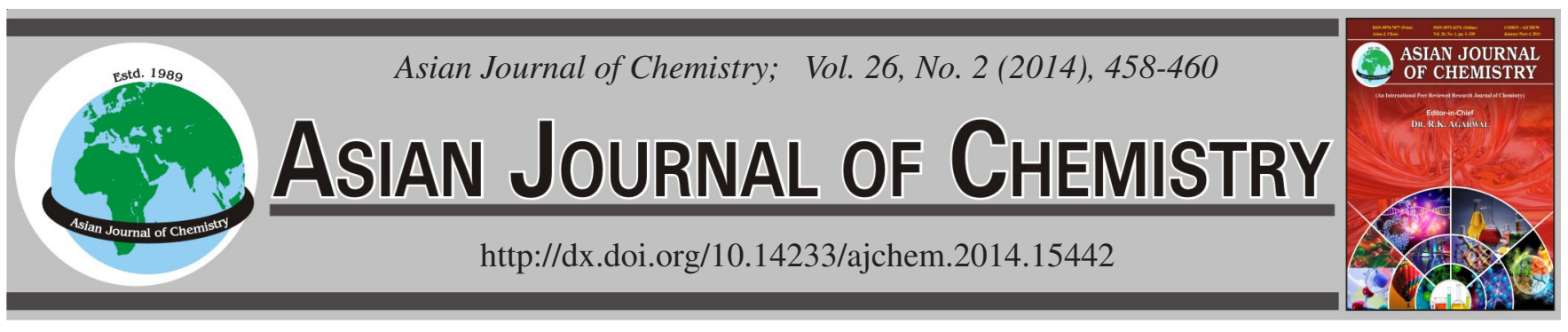

\title{
Detection of Antibacterial Compound of Avicennia marina Against Pathogens Isolated from Urinary Tract Infected Patients
}

\author{
A. Sheela Devi ${ }^{1, *}$, Johanna Rajkumar ${ }^{2}$ and T.K. BeEnish ${ }^{1}$
}

${ }^{1}$ Department of Biotechnology, Karpaga Vinayaga College of Engineering and Technology, Chinna Kolambakkam, Palayanoor Post, Madurantagam Taluk, Kanchipuram-603 308, India

${ }^{2}$ Department of Biotechnology, Rajalakshmi Engineering College, Thandalam, Chennai-602 105, India

*Corresponding author: Tel/Fax: +91 44 27565486; E-mail: sheeladevi.kvcet@ gmail.com

Received: 7 March 2013;

Accepted: 27 July 2013;

Published online: 15 January 2014;

AJC-14565

\begin{abstract}
Medicinal plants have been precious and important sources of natural products for the health of human beings and they have a great potential to produce new drugs. A number of mangroves and its associates possess new agrochemical products and biologically active compounds with medicinal value, which show biological activities such as antimicrobial properties. So they have capacity to treat diseases, because of its curative value. Also they are less toxic to humans and ecofriendly. A urinary tract infection (UTI) is an infection in the urinary tract. It almost certainly affects one-half of all people during their lifetimes. Bacteria are the most frequent cause of urinary tract infections. An antibacterial activity of leaves of Avicennia marina was evaluated against bacteria accountable for urinary tract infection and also antibacterial compound 1,3-benzodioxole,5,5-(tetrahydro- $1 H, 3 H$-furo [3,4-c]furan-1,4-diyl)bis-,[1s-(1 $\alpha, 3 \mathrm{a} \alpha, 4 \beta, 6 \mathrm{a} \alpha)]$ was isolated from leaf extract. Hence, the study suggests that $A$. marina can be used to treat urinary tract infection.
\end{abstract}

Keywords: Medicinal plants, Mangrove, Avicennia marina, Antibacterial compound, Urinary tract infection.

\section{INTRODUCTION}

An antimicrobial benefit of medicinal plants is ever more reported from different parts of the world ${ }^{1}$. It has been concluded that higher plants are prospective source for new antimicrobial agents ${ }^{2}$. Plant materials play a main role in primary health care as curative remedies in several developing countries $^{3}$. The plant product does not have a harmful effect in higher plants and animals including $\operatorname{man}^{4}$. India is a residence to a variety of conventional remedy that spread to a large extent for their raw drug materials ${ }^{5}$. There is a requirement to look back towards the traditional medicine which can serve as new remedial agent. Studies on antimicrobial activity of the plants to employ in systems require scientific testing. In current years, drug resistance to pathogens has been commonly and broadly accounted in literature ${ }^{6}$. Antimicrobials have significant medical value to treat antibiotic resistant microbial strains. Because of the side effects of antibiotics and antibiotic resistance microorganism scientists have paid notice to isolate biologically active compounds from plants used in herbal medicines? Mangroves have long been a source of astonishment for the layman and of interest for scientist. Mangroves are biochemically exclusive and producing a broad range of new products. They are long been used in folk medicine to treat diseases ${ }^{8}$. Mangrove and its associates contain biologically active anti- microbial compounds ${ }^{9}$. A. marina (Forssk.) is commonly known as gray mangrove and classified in the family of Avicenniaceae. It is frequently used to treat ulcers ${ }^{10}$. Urinary tract infection is the most common infection particularly for young and sexually energetic women. It is a severe health problem affecting millions of people per year and second most common type of infection in the body. Females are mainly prone to urinary tract infections than males. Additionally the female genital region supports the growth of numerous microorganisms. One woman in 5 develops urinary tract infection during her life time. Symptoms associated with urinary tract infection are painful burning sensation, urgent need to urinate, painful staining, disquiet in the middle of the lower abdomen, awakening from sleep at night to exceed urine and urethral pain etc. ${ }^{11}$. Therefore, in the present investigation the above mentioned plant extract was screened for their antibacterial activity against urinary tract infection pathogens. The objective of the present study is to isolate, purify and characterize the antibacterial compound.

\section{EXPERIMENTAL}

Collection and extraction of plant extract: The fresh and healthy leaves of Avicennia marina were collected from Pitchavaram, Thoothukudi district, TamilNadu, India. They were carefully identified and authenticated at Sidha Research 
Institute, Arumbakkam, Chennai, Tamil Nadu, India. After washing with distilled water, the leaves were shade dried, powdered and extracted using ethyl acetate. Fifty grams of plant powder was taken with $250 \mathrm{~mL}$ of solvent and kept in shaker for 1 day. After centrifuged at $5000 \mathrm{rpm}$, the solvent phase was separated and evaporated. The crude was stored at $40{ }^{\circ} \mathrm{C}$ and used for further studies ${ }^{12}$.

Isolation and Identification of pathogens: Midstream urine samples were inoculated into MacConkey Agar, Blood Agar, Cystine Lysine electrolyte deficient media (CLED) agar, SDA and incubated at $37{ }^{\circ} \mathrm{C}$ for $24 \mathrm{~h}$. The bacterial colonies were identified by biochemically and morphologically. The biochemical tests are gram staining, motility test, catalase test, oxidase test, indole test, methyl red (MR) test, voges-proskauer (VP) test, citrate utilization test, triple sugar iron agar test, urease test in urea broth and oxidation fermentation (OF) test.

The identified strains were two Gram-positive (Staphylococcus aureus and Enterococci) and five gram-negative (Escherichia coli, Klebsiella pneumoniae, Pseudomonas aeruginosa, Enterobacter and Proteus vulgaris) bacterium. Out of these we have selected Staphylococcus aureus, Escherichia coli, Pseudomonas aeruginosa and Klebsiella pneumonias for present study.

The organisms were sub-cultured on Mueller Hinton Agar medium (MHA) (HI MEDIA, Mumbai, India), incubated at $37^{\circ} \mathrm{C}$ for $24 \mathrm{~h}$ and stored at $4{ }^{\circ} \mathrm{C}$ in the refrigerator to maintain stock culture for further studies.

Determination of antibacterial activity: The crude ethyl acetate extract of $A$. marina was subjected to antimicrobial assay using disc diffusion method ${ }^{13} .20 \mathrm{~mL}$ of nutrient agar was poured into sterile petridishes. The test cultures were swabbed on the top of the solidified media and allowed to dry for $10 \mathrm{~min}$. The tests were conducted with the crude ethyl acetate extract. The loaded discs were placed on the surface of the medium and left for $0.5 \mathrm{~h}$ at room temperature for compound diffusion. Zone of inhibition was recorded in millimetres and the experiment was repeated in three replicates.

\section{Separation and identification of antibacterial compound from ethyl acetate extract}

Fractionation of the ethyl acetate extract: Activated silica gel (60-120 mesh) was packed onto a glass column (450 $\mathrm{mm} \times 40 \mathrm{~mm}$ ) using $n$-hexane solvent. The ethyl acetate extract was loaded on top of the silica gel and eluted stepwise using suitable solvents. About 41 fractions measuring $100 \mathrm{~mL}$ each were collected and concentrated by using the rotary evaporator.

Thin-layer chromatography (TLC): An aliquot of all the concentrated fractions were loaded on the activated silica gel TLC plates $(20 \mathrm{~cm} \times 20 \mathrm{~cm})$. The plates were developed using proper solvents and spots were located by exposing the plate to iodine fumes ${ }^{14}$. Fraction showing the same number of spots with similar $R_{f}$ values on the TLC plate were pooled into 5 fractions. All the 5 pooled fractions were tested for antibacterial activity by agar well diffusion method.

Further purification of the bioactive fraction: Since fraction three (Fr. 3) obtained from the first step of column chromatography showed high antibacterial activity, it was selected for further purification. Bioactive Fr. 3 was further purified using a silica gel (60-120 mesh) column (450 $\mathrm{mm} \times$
$20 \mathrm{~mm}$ ). The column was eluted step-wise with suitable solvents. About 15 fractions measuring $100 \mathrm{~mL}$ each were collected and concentrated on a rotary evaporator. An aliquot of all the fractions was loaded on the TLC plate and fraction showing similar $\mathrm{R}_{\mathrm{f}}$ values were pooled into 8 sub-fractions (Fr. 3.1-Fr. 3.8). These 8 fractions were tested for antibacterial activity.

Sub-fraction five (Fr. 3.5) obtained from second-step chromatography showed high antibacterial activity and was hence selected for further purification. Bioactive sub-fraction five (Fr. 3.5) was further purified on a silica gel (100-200 mesh) column $(600 \mathrm{~mm} \times 15 \mathrm{~mm})$. The column was eluted step-wise with suitable solvents. About 21 fractions measuring $25 \mathrm{~mL}$ each were collected and concentrated. Fractions having similar $\mathrm{R}_{\mathrm{f}}$ values on the TLC plate were pooled into 6 sub-fractions (Fr. 3.5.1-Fr. 3.5.6). Among these, fraction number five (Fr. 3.5.5) obtained from third-step chromatography showed high antibacterial activity and a single spot in the TLC profile. Hence this concentrated pure fraction was subjected to gas chromatography-mass spectrometry (GC-MS).

Identification and characterization of the active antibacterial compound by GC-MS: GC-MS was used for identification and characterization of the molecule. $0.20 \mu \mathrm{L}$ of purified concentrated fraction was loaded in GC injector using 10 $\mu \mathrm{L}$ syringe. The GC-MS (Agilent 6890) was fitted with EMV mode. Helium was used as the carrier gas at a flow rate of $1 \mathrm{~mL} / \mathrm{min}$. The total runtime of the sample was maintained for $35 \mathrm{~min}$. The compound of interest was identified by the gas chromatographic image. The gas chromatogram was plotted with retention time on $\mathrm{x}$-axis and abundance of compound on $y$ axis. The retention time and abundance of each peak was found out and the longest peak which has highest abundance was selected as the compound of interest. The compound name and structure was identified by comparing the retention time from the gas chromatogram with the molecular weight obtained from the mass spectrometry and analysing these values with compounds in GC-MS database.

\section{RESULTS AND DISCUSSION}

The ethyl acetate extract of A. marina leaves showed significant zone of inhibition against one "gram positive" bacteria-Staphylococcus aureus and three "gram-negative" bacterium-Escherichia coli, Pseudomonas aeruginosa and Klebsiella pneumonias (Fig. 1).

The plant extract exhibited more inhibition for Staphylococcus aureus than for other bacterial species taken for the study. Our data showed that, antibacterial extract from A.marina appear to be more inhibitory to gram-positive organism than to gram-negative types. Unlike gram-positive bacteria, the chief component of outer layer of gram-negative bacteria is lipopolysaccharide. It present along with proteins and phospholipids. The outer layer might hinder contact of antibacterial compound to the peptidoglycan layer of the cell wall $^{15}$. Extracts from mangroves and its associates are reported to possess medicinal properties and they are very active against human and plant pathogens ${ }^{16}$. Identification and characterization of the molecule was done by GC-MS.

Identification of active antibacterial compound: The most active antibacterial compound was identified as 1,3- 


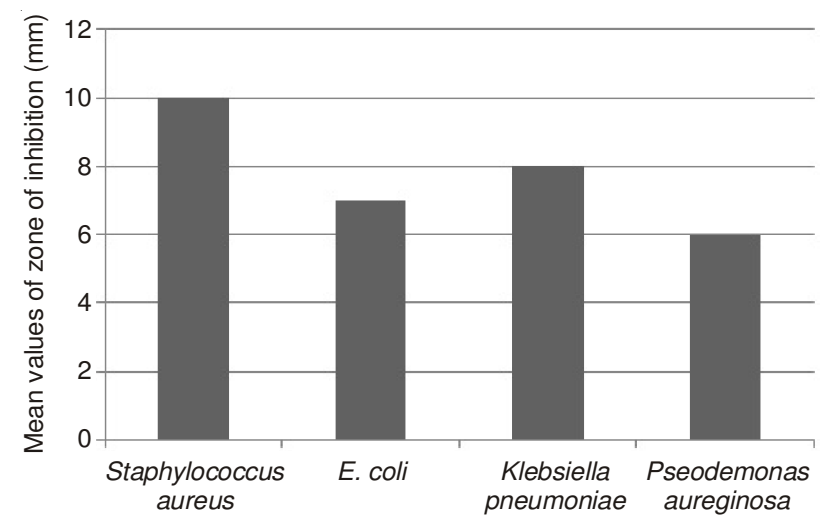

Fig. 1. Antibacterial activities of ethyl acetate extract of $A$. marina leaves (each zone value represents the mean diameter of three replicates)

benzodioxole,5,5-(tetrahydro- $1 H, 3 H$-furo[3,4-c]furan-1,4diyl)bis-,[1s-(1 $\alpha, 3 \mathrm{a} \alpha, 4 \beta, 6 \mathrm{a} \alpha)]$.

The compound has showed highest abundance and the three long peaks with retention time 27.66, 27.90 and 28.13 was the same compound; it was identified from GC-MS library.
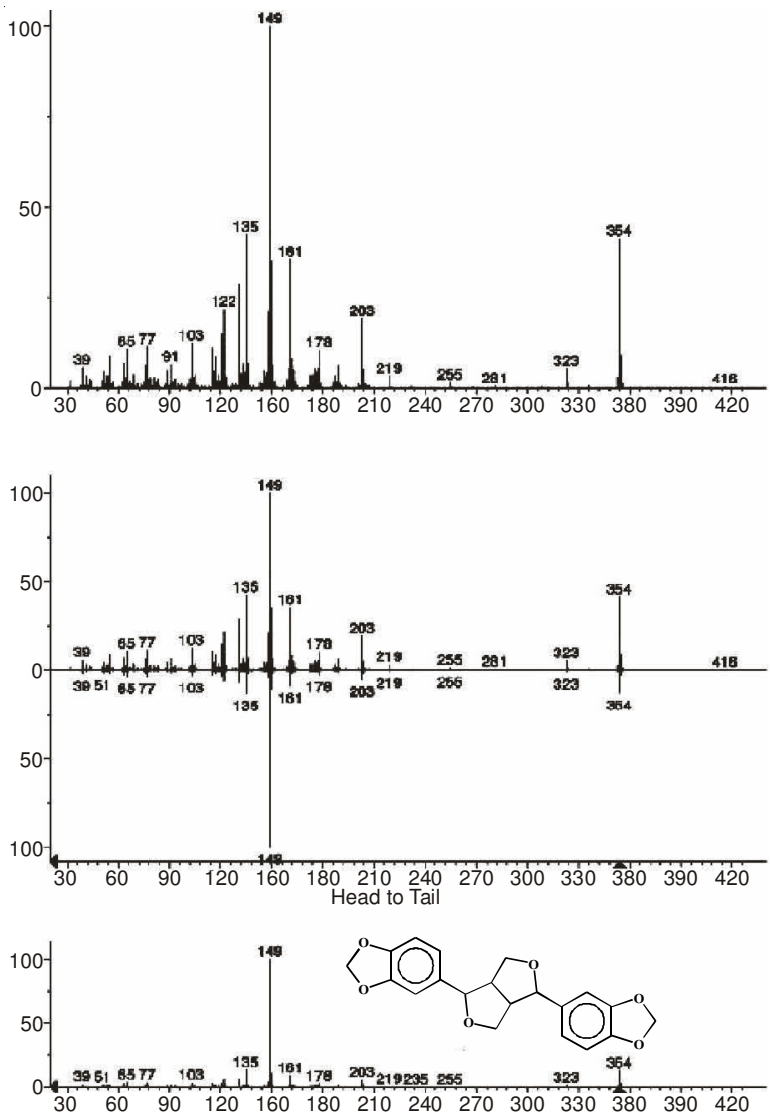

Fig. 2. Mass spectrum of peak with retention time 27.66, 27.90 and 28.13

Characterization of the compound: The molecular weight was determined as 354 kilo Dalton. The structure of the compound was found out by comparing retention time with molecular weight from the GC-MS library.

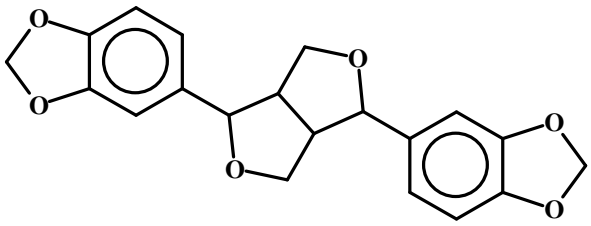

Fig. 3. Structure of active compound

\section{Conclusion}

The ethyl acetate extract of $A$. marina leaves appear to be more inhibitory to gram-positive organism than to gramnegative types. Antibacterial activity-guided fractionation of the leaves of plant extract yielded a bioactive compound. The structure of the isolated antibacterial compound was elucidated with spectroscopic technique and identified as 1,3-benzodioxole, 5,5-(tetrahydro- $1 \mathrm{H}, 3 \mathrm{H}$-furo[3,4-c]furan-1,4-diyl)bis-, [1s $(1 \alpha, 3 \mathrm{a} \alpha, 4 \beta, 6 \mathrm{a} \alpha)]$. It showed bactericidal activity against a broad range of bacteria responsible for urinary tract infection. Hence it has an enormous potential and are worth exploring for pharmaceutical uses.

\section{ACKNOWLEDGEMENTS}

The authors thank Prof. Dr. Niranjali Devaraj, Department of Biochemistry, Chennai University and Prof. Dr. Ramalingam, Department of Biotechnology, Anna University, Chennai for their valuable suggestions.

\section{REFERENCES}

1. V.K. Saxena and R.N. Sharma, Fitoterapia, 70, 64 (1999).

2. L.A. Mitscher, S. Drake, S.R. Golloapudi, S.K. Okwute, V. Siddaiah and C.V. Rao, J. Nat. Prod., 50, 1025 (1987).

3. A. Sokmen, B.M. Jones and M. Erturk, J. Ethnopharmacol., 67, 79 (1999).

4. C. Chitravadivu, S. Manian and K. Kalaichelvi, Middle-East J. Sci. Res., 4, 144 (2009).

5. R. Santhi, C. Alagesaboopathi and M. Rajasekara Pandian, Adv. Plant Sci., 19, 371 (2006)

6. M.E. Mulligan, K.A. Murray-Leisure, B.S. Ribner, H.C. Standiford, J.F. John, J.A. Korvick, C.A. Kauffman and V.L. Yu, Am. J. Med., 94, 313 (1993).

7. T. Essawi and M. Srour, J. Ethnopharmacol., 70, 343 (2000).

8. W.M. Bandaranayake, Mangroves Salt Marshes, 2, 133 (1998).

9. M.I. Okeke, C.U. Iroegbu, E.N. Eze, A.S. Okoli and C.O. Esimone, J. Ethnopharmacol., 78, 119 (2001).

10. M. Subashree, P. Mala, M. Umamaheswari, M. Jayakumari, K. Maheswari, T. Sevanthi and T. Manikandan, Int. J. Curr. Res., 1, 016(2010).

11. J.E. Fowler Jr., Urinary Tract Infections in Women, Urologic Clinics of North America, pp. 673-683 (1986).

12. N.B. Dhayanithi, T.T. Ajith Kumar, R.G. Murthy and K. Kathiresan, Asian Pacific J. Trop. Biomed., 2, S1892 (2012).

13. A.W. Bauer, W.M. Kirby, C. Sherris and M. Turck, Am. J. Clin. Pathol., 45, 493 (1966).

14. A. Amoozadeh and H. Zavvar Mousavi, Asian J. Chem., 20, 5873 (2008).

15. P.D. Abeysinghe, Indian J. Pharm. Sci., 72, 167 (2011).

16. S. Ravikumar, M. Gnanadesigan, P. Suganthi and A. Ramalakshmi, Int. J. Med. Med. Sci., 2, 94 (2010). 